\title{
Consumo de cine en Colombia: estimación de sus determinantes ${ }^{*}$
}

\section{Cinema consumption in Colombia: an economic approach}

\author{
Nora Elena Espinal-Monsalve \\ Departamento de Economía, Universidad Nacional de Colombia, Medellín, Colombia \\ nespinal@unal.edu.co \\ https://orcid.org/0000-0003-2777-7410
}

Andrey David Ramos-Ramírez ${ }^{2}$

Departamento de Economía, Universidad Nacional de Colombia, Medellín, Colombia adramosr@unal.edu.co

https://orcid.org/0000-0002-5795-3190

Isabel Cristina Balbín-Espinal ${ }^{3}$

Facultad de Ciencias, Universidad Nacional de Colombia, Medellín, Colombia

icbalbine@unal.edu.co

https://orcid.org/0000-0002-9907-9067

Jorge Echavarría-Carvajal ${ }^{4}$

Departamento de Estudios Filosóficos y Culturales, Universidad Nacional de Colombia, Medellín, Colombia jechavar@unal.edu.co

https://orcid.org/0000-0001-9947-561X

Recibido: $27-11-18$

Aprobado: $27-08-19$

\footnotetext{
* Este artículo es resultado del proyecto de investigación "Análisis comparativo de los determinantes de la participación cultural en Colombia: el caso de las artes escénicas y el Cine" realizado por los autores, entre agosto de 2017 y junio de 2018, con financiación de la "Convocatoria Nacional de Proyectos para el Fortalecimiento de la Investigación, Creación e Innovación de la Universidad Nacional de Colombia 2016-2018".

1 Doctora en Economía y Empresa.

2 Magíster en Ciencias Económicas.

3 Estadística.

4 Magíster en Estética.
} 


\section{Resumen}

En este artículo se analizan los determinantes de la asistencia y frecuencia de asistencia a cine en Colombia. Utilizando los datos de la Encuesta de Consumo Cultural 2014, se estiman modelos de selección de Heckman que permiten detectar y corregir los sesgos de selección que se pueden presentar en el problema de investigación. Los resultados indican que, aunque esta actividad se enmarca en un modelo de consumo cultural de masas, el capital cultural y los ingresos siguen siendo determinantes importantes en las decisiones de consumo de los individuos. Factores como la edad, el estado civil y la ocupación también resultaron relevantes. El presente trabajo contribuye a la literatura aplicada en el país en la medida en que permite un conocimiento más amplio del comportamiento del consumidor de cine.

Palabras clave: comportamiento cultural; asistencia al cine; capital cultural; selección muestral.

Clasificación JEL: C35, D11, Z11

\section{Abstract}

This article analyzes the determinants of attendance and frequency of assistance to cinema in Colombia. Using the data from the 2014 Cultural Consumption Survey, Heckman selection models are estimated to detect and correct selection biases that may arise in the research problem. The results indicate that although this activity is part of a model of mass cultural consumption, cultural capital and income, they remain important determinants in the consumption decisions of individuals. Factors such as age, marital status and occupation were also relevant. This work contributes to the applied literature in the country, to the extent that it allows a broader behavioral knowledge of the cinema consumer.

Keywords: Cultural behavior; Cinema Attendance; Cultural Capital; Sample Selection.

¿Cómo citar este artículo? / How to quote this article?

Espinal-Monsalve, N. E., Ramos-Ramírez, A. D., Balbín-Espina, I. C. y Echavarría-Carvajal, J. (2020). Consumo de cine en Colombia: estimación de sus determinantes. Sociedad y economía, (39), 57-79. https://doi.org/10.25100/sye.voi39.7200 


\section{Introducción}

El consumo de bienes y servicios culturales se define como un proceso social en el que se realiza la apropiación, recepción y uso de los bienes o servicios producidos en el sector cultural (García y Piedras, 2000). El análisis de las decisiones de consumo cultural ha sido objeto de reflexión de diferentes disciplinas, especialmente de la economía y la sociología, reconociendo que los individuos eligen los bienes por consumir no solamente con base en la racionalidad de la maximización de la utilidad, sino también como una manifestación de las identidades individuales, familiares y sociales, que se constituyen a su vez en parte de un proceso de reproducción social (Bourdieu, 1984; Thompson, 1990).

Los planteamientos desde la economía sugieren que el gusto por los bienes culturales es determinado por fenómenos sistemáticos y que es posible explicar el comportamiento de los consumidores a partir de la teoría microeconómica. El enfoque de la adicción racional, propuesto por Stigler y Becker (1977) y Becker y Murphy (1988), por ejemplo, plantea que los gustos son exógenos, estables e idénticos entre los individuos. Las diferencias en los niveles de consumo cultural no responden a cambios en las preferencias, sino a diferencias en factores como el precio, la disponibilidad de tiempo o el capital cultural, entendiendo el último como una faceta del capital humano que otorga a los individuos la capacidad de interpretar los valores simbólicos, estéticos e históricos que incorporan los bienes culturales (Ateca-Amestoy, 2009). Los consumidores generan una adicción por estos bienes debido a que, con cada experiencia de consumo, acumulan conocimientos específicos en forma de capital cultural, que incrementan determinísticamente la capacidad de apreciación y el nivel de satisfacción que obtendrían en las experiencias futuras.

El enfoque del aprendizaje en el consumo, por su parte, difiere de la adicción racional en el sentido que endogeniza el proceso de formación del gusto y permite diferencias en los esquemas individuales de preferencias. En tal caso, el gusto por los bienes culturales se construye a través de un proceso secuencial de aprendizaje en el cual el individuo realiza un contraste subjetivo entre sus expectativas, con respecto a la satisfacción que espera obtener con cada experiencia, y el nivel de satisfacción real que obtiene en el acto del consumo; el gusto se forma como un cúmulo de experiencias positivas que conllevan a una revisión de las expectativas y un incremento de los niveles de consumo en el futuro (Lévy-Garboua y Montmarquette, 1996). El aspecto común que comparten los dos enfoques económicos expuestos es que el consumo presente implica acumulación de conocimientos, experiencia y habilidades $y$, en este sentido, determina el nivel de satisfacción que se espera obtener en el futuro, bien sea porque sistemáticamente se genera una adicción o porque los individuos ajustan sus expectativas (Throsby, 1994; Castiglione e Infante, 2016).

Los estudios, desde la sociología, se han enfocado en establecer las relaciones entre el origen social y los hábitos culturales de los individuos, es decir, cómo los estilos de vida y los consumos culturales se ven condicionados por las características de la estructura social (Herrera-Usagre, 2010). En esta línea, Bourdieu (1984) plantea que existe una correlación entre el estrato social y los hábitos culturales, de manera que los individuos que pertenecen a las clases sociales altas tienen gustos y consumos de la "alta cultura", mientras que los individuos de las clases sociales bajas tienen consumos de la "cultura popular". Tal modo de diferenciación crea una estructura jerárquica de los gustos y los estilos de vida que reproduce el esquema de jerarquías sociales. Al respecto, Alonso (1995) plantea que el consumo cultural tiene valores sociales que lo constituyen en un elemento de reproducción social, a saber: el valor de identidad (Bocock, 1995), entendiendo que al realizar un consumo cultural el individuo se identifica con un modelo de vida, el valor de expresividad (Jenks, 1993), en el sentido de que el consumo cultural es una expresión de la personalidad, y el valor de reflexividad (Varela, 1988) que representa la idea de auto organización social.

En la definición de los patrones de consumo cultural no solo intervienen las motivaciones individuales, como lo plantean la economía y la sociología, sino también aspectos relacionados 
con los mecanismos de producción y distribución de los productos culturales. Según Lazcano y Landabidea (2010), desde finales de la Segunda Guerra Mundial hasta la crisis de los 70 y la postcrisis de los 80, la cultura de masas se convirtió en el modelo de consumo cultural hegemónico. Este modelo impulsó un proceso de industrialización de la producción cultural, lo que generó la producción en cadena de objetos culturales estandarizados y se ocupó de que los soportes técnicos fueran puestos a disposición del gusto cultural de las clases medias. El proceso es más evidente en las industrias culturales que en las artes escénicas o el patrimonio cultural, lo cual conlleva a diferencias en los factores determinantes del consumo cultural de tales actividades.

En efecto, la literatura empírica ha mostrado que el consumo de artes escénicas y de patrimonio cultural es predominantemente elitista, influenciado fuertemente por las experiencias y el gusto adquiridos a través de la acumulación de capital cultural (Borgonovi, 2004; Falk y Falk, 2011; Gómez y Espinal, 2016; Ramos, 2017; entre otros). En contraste, en el consumo de productos de las industrias culturales, como el cine, los estudios han mostrado que los requerimientos de capital cultural para disfrutar de esta actividad son menores. La mayoría de los estudios aplicados en contextos de países como Estados Unidos o Inglaterra -que abarcan un largo periodo de tiempo-ponen de manifiesto que, aunque ir a cine está en correlación inversa a la clase social, los ingresos o la educación, tales correlaciones son débiles. La influencia de otro tipo de factores como la edad, la etnia o el género han mostrado ser más fuertes (Hinojosa, 2016).

Uno de los estudios pioneros sobre públicos de cine fue realizado por Katz y Lazarsfeld (1955) quienes encontraron que, en Illinois, Estados Unidos, ir a cine era una actividad realizada por la población joven, y que las mujeres actuaban más como líderes de opinión en la selección de las películas. Por su parte, Abrams (1950) estudió el público asistente a cine en Gran Bretaña durante tres años: 1946, 1947 y 1948. El autor encontró que ir a cine era una actividad regular y de relajación semanal, y que no existían diferencias significativas en los hábitos de los hombres y las mujeres; además, la clase trabajadora, los jóvenes y los solteros eran quienes más asistían a cine. Tudor (1974) destaca que existen ciertos factores socioculturales que afectan la decisión de asistir a cine; por ejemplo, ir a cine se refiere más a una institución social que a una experiencia individual. La gente va a cine en grupo porque motiva temas de conversación, facilita los contactos informales entre personas, promueve reuniones, citas, amistades u otras relaciones interpersonales o grupales (Hinojosa, 2016).

Aunque se registran estudios sobre el cine desde la primera mitad del siglo pasado, estos fueron realizados en el marco de la comunicación y la sociología. Los primeros trabajos elaborados desde la economía aparecen en los albores del siglo XXI. Tales estudios son de dos tipos: $i$ ) los que utilizan datos macroeconómicos para predecir el número de visitantes a cine y ii) los que utilizan datos a nivel microeconómico para cuantificar la influencia de los factores sociodemográficos y del capital cultural en la decisión de asistir a la actividad. En el primer tipo de estudios se destaca el de Cameron (1999), quien estimó un modelo de adicción racional usando una ecuación de demanda de cine para el Reino Unido empleando datos recogidos entre 1956 y 1983. El autor, al aplicar Mínimos Cuadrados Ordinarios, encontró que el consumo pasado influye sobre el nivel de consumo presente de la sociedad; sin embargo, al estimar el modelo por el método de Variable Instrumental, la evidencia sobre el cumplimiento del enfoque resultó débil. Sisto y Zanola (2010) también analizaron el componente adictivo del cine desde un punto de vista macroeconómico, utilizando datos de 12 países europeos en el periodo 1989 - 2004. Los resultados aportan evidencia a favor de un modelo de demanda de cine que enfatiza en la influencia del consumo pasado sobre el consumo presente y futuro; adicionalmente, encontraron que la demanda responde elásticamente a los precios en el largo plazo.

Los estudios a nivel microeconómico validan los hallazgos de los estudios de públicos realizados desde la comunicación. Katsuura (2008) analiza la decisión de asistencia a varias actividades culturales en Japón, entre ellas el cine, usando datos del 2001. En el trabajo se encontró que las 
mujeres y la población joven tienen una mayor probabilidad de asistir a cine; además, la elasticidad del consumo de esta actividad con respecto al ingreso es baja en comparación con lo calculado para el caso de la asistencia a conciertos de música clásica o teatro. El nivel educativo también arrojó un efecto positivo sobre la asistencia a cine, aunque es menor en comparación con el de las actividades consideradas de alta cultura. Ateca-Amestoy (2010), por su parte, analizó los determinantes de la asistencia y la frecuencia de asistencia a cine en España usando datos para 2002 - 2003. En su estudio se encontró que, aunque las mujeres tienden a participar más que los hombres, el sexo no tiene efectos significativos sobre la intensidad de las visitas; similarmente, los individuos con niveles más altos de educación asisten a cine con una mayor probabilidad, aunque esta variable no determina la frecuencia de asistencia. El ingreso influye positivamente tanto en la participación como la frecuencia con que los individuos asisten.

En Colombia existe un auge reciente de análisis empíricos en los que se analizan los determinantes de participación en diferentes actividades culturales como las artes escénicas (Aguado, 2011; Gómez y Espinal, 2016), museos (Andrade, 2016; Ramos, 2017), bibliotecas (Palma, Aguado y Osorio, 2014; Graziano, Escobar, Angulo y Mercado, 2017; Ramos, 2017), música grabada (Bermúdez, Medina y Aguado, 2016), lectura de periódicos (Graziano et al., 2017; Aguado, Lazzaro, Palma y Osorio, 2018), entre otros. En el caso del cine, se encontraron los estudios de Espinosa y Toro (2016) y Graziano et al. (2017) quienes analizan la participación en esta actividad en Cartagena y en la Región Atlántica, respectivamente. El presente trabajo contribuye a la literatura aplicada en el país en la medida en que es un estudio que utiliza datos a nivel nacional. Esto permite el conocimiento más amplio del consumidor de cine y establecer diferencias interregionales; además, se aplica una metodología no utilizada hasta el momento en la modelación de las decisiones de participación cultural en Colombia.

El objetivo del presente artículo es analizar los determinantes de la asistencia y frecuencia de asistencia a cine en Colombia, aplicando modelos de selección de Heckman para variable dependiente categórica ordenada. Utilizando los datos de la Encuesta de Consumo Cultural (ECC) aplicada por el Departamento Administrativo Nacional de Estadística (DANE) en 2014 (ECC2014), se estiman las ecuaciones de participación que permiten cuantificar el efecto ceteris paribus de las características sociodemográficas y del capital cultural sobre las decisiones de consumo de los individuos. El enfoque metodológico de los modelos de selección tiene la ventaja de corregir el posible sesgo de selección que aparece cuando la muestra empleada en la estimación no es aleatoria, lo cual podría suceder en el problema de investigación debido a que la frecuencia de asistencia solamente es observable cuando el individuo efectivamente decide asistir.

El artículo se compone de cuatro apartados incluyendo la presente introducción. En el segundo apartado se describen las fuentes de información, las variables y el enfoque metodológico adoptado en el análisis empírico. En el tercer apartado se describen los resultados obtenidos en los modelos econométricos estimados. En el cuarto apartado se discuten los resultados, se presentan las conclusiones y se debate acerca de las perspectivas de investigación en la línea de consumo cultural.

\section{Metodología}

En el apartado metodológico se describen los datos y variables empleados en el análisis empírico, así como los modelos econométricos estimados que permiten obtener el efecto ceteris paribus de las variables explicativas sobre las decisiones de asistencia y frecuencia de asistencia a cine en el año 2014.

\subsection{Datos y variables}

Los datos usados en la investigación provienen de la ECC, aplicada por el Departamento Administrativo Nacional de Estadística (DANE) en $2014^{5}$. La ECC se realiza cada dos años con el propósito

5 Disponible en: http://microdatos.dane.gov.co/index. php/catalog/345/get_microdata. 
de indagar a los individuos mayores de 12 años por las decisiones de consumo cultural en los componentes de presentaciones y espectáculos, espacios culturales, publicaciones, audiovisuales y música, práctica cultural y hábitos de lectura. En 2014 fueron encuestados 26.302 individuos en 8.623 hogares ubicados en las cabeceras municipales de las regiones Atlántica, Oriental, Central, Pacífica, Orinoquia-Amazonía y Bogotá.

La ECC2014 contiene información específica sobre la asistencia a cine en los 12 meses anteriores a la aplicación del cuestionario, así como sobre la frecuencia de asistencia para quienes responden afirmativamente. Dos tipos de variables dependientes de interés para este estudio se generan a partir de la información. La primera es una variable dicotómica que captura la decisión de asistencia; mientras que la segunda es una variable categórica ordenada que clasifica a los asistentes en Ocasionales (asisten una o dos veces al año), Frecuentes (asisten una vez cada 3 meses) y Muy frecuentes (asisten por lo menos una vez al mes) ${ }^{6}$. La ECC2014 también recoge información sobre las características personales, socioeconómicas, ubicación geográfica y capital cultural que influyen en el comportamiento de los individuos respecto al consumo de cine.

Una de las variables socioeconómicas que se incluyen en el presente estudio es el nivel de ingresos del individuo. Los estudios aplicados a nivel nacional e internacional muestran que el ingreso tiene una correlación positiva con la participación cultural (Borgonovi, 2004; AtecaAmestoy, 2009; Fernández-Blanco y PrietoRodríguez, 2009; Gómez y Espinal, 2016; Ramos, 2017; entre otros). Esto indica la existencia de restricciones monetarias que condicionan el acceso de la población a los bienes y servicios culturales. Ingresos altos implican una mayor capacidad para pagar por las actividades de ocio, y si los bienes y servicios culturales son bienes

6 En la ECC 2014 se establecen 5 categorías para la frecuencia de asistencia: 1 . Una vez al año, 2. Una vez cada seis meses, 3. Una vez cada tres meses, 4. Una vez al mes y 5. Por lo menos una vez a la semana. Para facilitar el análisis, en este trabajo se redujeron el número de categorías según criterios del investigador. normales, la teoría económica predice una elasticidad de ingreso positiva. En los estudios realizados en Colombia, como en los casos de Aguado (2011), Palma et al. (2014), Bermúdez et al. (2016), Espinosa y Toro (2016) y Gómez y Espinal (2016), se considera el estrato socioeconómico como variable explicativa que da cuenta de la posición socioeconómica de los individuos y que permite asociar la participación cultural con patrones de segregación espacial; tal variable no es considerada en el presente estudio debido a que no fue incluida en la ECC 2014, lo que constituye una limitación.

Respecto al capital cultural, por la disponibilidad de información, se mide en tres dimensiones que han sido empleadas tradicionalmente en la literatura (Ateca-Amestoy, 2009): $i$ ) el nivel de educación formal, ii) la participación activa en prácticas de audiovisuales, talleres de audiovisuales y actividades lúdicas, y iii) la participación como asistente o consumidor de otras actividades culturales (sitios de interés histórico, música, televisión, museos, artes escénicas y conciertos). En la Tabla A-l del Anexo se describen detalladamente cada una de las variables consideradas en el análisis.

\subsection{Modelos de selección muestral}

En el análisis de la frecuencia de asistencia a cine es posible que la muestra utilizada en la estimación no sea aleatoria, debido a que la observación de la variable dependiente está condicionada a la decisión previa de asistencia. La literatura estadística ha mostrado que, en presencia de selección muestral, los estimadores de los modelos probit ordenados son sesgados e inconsistentes (Heckman, 1979; De Luca y Perotti, 2011). Para superar tal limitación, en este trabajo se estiman modelos de selección muestral para variable dependiente categórica ordenada en los cuales se modela simultáneamente la decisión de asistencia (Ecuación de selección) y la intensidad con que los individuos asisten (Ecuación de resultado).

Las ecuaciones estructurales del modelo son (Greene y Hensher, 2010): 


$$
\begin{aligned}
& y_{1}^{*}=x_{1}^{\prime} \beta_{1}+\varepsilon_{1} \text { (Ecuación de selección) (1) } \\
& y_{2}^{*}=x_{2}^{\prime} \beta_{2}+\varepsilon_{2} \text { (Ecuación de resultado) (2) }
\end{aligned}
$$

donde $y_{1}^{*}$ es una variable latente que es observada parcialmente a través de la decisión observable de asistencia $y_{1}$, con $y_{1}=1$ para los individuos que asistieron a cine y $y_{1}=0$ en otro caso; $y_{2}{ }^{*}$ es otra variable latente relacionada con la frecuencia de asistencia, que puede tomar el valor observable $y_{2}=j$ de un conjunto de alternativas ordenadas $J$ cuando $y_{1}=1 ; x_{1}$ y $x_{2}$ son las variables explicativas asociadas a la ecuación de selección y a la ecuación de resultado respectivamente (ver apartado de variables); $\beta_{1}$ y $\beta_{2}$ son los coeficientes asociados a $x_{1}$ y $x_{2}$ respectivamente; $\varepsilon_{1}$ y $\varepsilon_{2}$ son los términos de perturbación aleatorios de cada una de las ecuaciones que describen el modelo. Este modelo asume que los componentes de error de las ecuaciones siguen una distribución normal bivariada con un coeficiente de correlación $\rho, \rho \neq 0$ donde implica que existen sesgos de selección y el análisis tradicional para modelo con variable dependiente ordenada genera resultados inconsistentes (Greene y Hensher, 2010).

En la literatura es posible encontrar aplicaciones en las que los autores han "corregido" la selección muestral siguiendo la lógica del modelo de Heckman en dos etapas, es decir, construyendo el ratio de Mills con la estimación de un modelo probit ordenado y agregándolo a la ecuación de resultado. Sin embargo, tal procedimiento es adecuado cuando el modelo es lineal y, evidentemente, la segunda etapa en este modelo no lo es. En tal caso, el procedimiento de estimación consiste en maximizar la siguiente función de log - verosimilitud (Greene y Hensher, 2010):

$$
\ln L=\sum_{y 1=0} \log \Phi\left(-x_{1}^{\prime} \beta_{1}\right)+\sum_{y 1=1} \sum_{j=0}^{I} m i j \log \left[\Phi 2\left(\mu_{j}-x_{2}^{\prime} \beta_{2}, x_{1}^{\prime} \beta_{1} \rho\right)-\Phi 2\left(\mu_{j-1}-x_{2}^{\prime} \beta_{2}, x_{1}^{\prime} \beta_{1} \rho\right)\right](3)
$$

donde $m_{i j}=1$ si $y_{2}=j, \Phi_{2}($.) es la acumulada de la función de distribución normal bivariada con vector de medias cero, $\Phi\left(\right.$.) es la acumulada de la distribución normal estándar, y $\mu_{j}$ es tal que $y_{2}=j$ si $\mu_{j-1}<y_{2}^{*}<\mu_{j}$.

La adecuada identificación de los parámetros del modelo requiere que la ecuación de selección contenga al menos una variable explicativa que no esté en la ecuación de resultado (De Luca y Perotti, 2011). La importancia de imponer esta restricción de exclusión se ha discutido ampliamente en la literatura sobre modelos de selección muestral y modelos de elección discreta, especialmente en Meng y Schmidt (1985), Keane (1992) y Lee (1995). En este trabajo se utilizan los resultados del modelo de Heckman No Restringido (HNR) y consideraciones teóricas para identificar la restricción de exclusión apropiada para estimar el modelo de Heckman Restringido (HR); los resultados que se interpretan son los del modelo HR. Una condición adicional para la identificación es la inclusión de al menos una variable continua en las dos ecuaciones (De Luca y Perotti, 2011), que en este caso es el número de personas en el hogar. La estimación del modelo se realiza en STATA con el comando heckoprobit.

\section{Resultados}

Las mediciones realizadas por la ECC en los años 2010, 2012 y 2014 indican que el porcentaje de asistentes a cine en Colombia ha crecido levemente. En 2010, el 29,0\% de los individuos mayores de 12 años asistía a tal actividad, el porcentaje se incrementó a 31,1\% en 2012 y 33,8\% en 2014. Este primer hallazgo indica que los comportamientos de la población respecto al cine están cambiando en el país, por lo tanto, conocer los factores que determinan tal comportamiento cultural es información útil para orientar las estrategias de política que potencien el acceso de un mayor número de personas y permitan un crecimiento mayor de las tasas de asistencia. A continuación, se presentan 
los resultados del análisis empírico realizado solamente con los datos de la ECC2014, es decir, que el estudio es de corte transversal.

\subsection{Estadísticas descriptivas}

En la Tabla 1 se presenta la caracterización de los grupos de asistentes, no asistentes y muestra completa según las variables explicativas descritas en la Tabla A-l, así como el valor $p$ de las pruebas Chi-Cuadrado de Pearson realizadas para probar la hipótesis nula de independencia entre la asistencia a cine y cada una de las variables explicativas. Los resultados de las pruebas indican (con un nivel de significancia del 5\%) que la distribución de la asistencia a cine depende de los niveles que tomen las variables explicativas consideradas. En efecto, al comparar las características del grupo de asistentes con respecto a la muestra completa se observa una mayor proporción de asistentes con edad entre los 12-24 años, solteros, mestizos o blancos, estudiantes, con ingresos superiores a $\$ 700.000$, residentes en Bogotá, con educación superior o de posgrado, que realizan actividades lúdicas y que asisten o consumen otras actividades del sector cultural. En este sentido, la evidencia preliminar sugiere que tales características tienen una influencia positiva sobre la decisión de asistir a cine.

\subsection{Modelos estimados}

Las percepciones preliminares derivadas del análisis descriptivo bivariado se verifican formalmente con la estimación de los modelos econométricos. En la Tabla A-2 del Anexo se reportan los resultados del modelo HNR. Con base en la evidencia del modelo se determina que la restricción de exclusión a aplicar en este trabajo consiste en omitir la Ocupación de la ecuación de resultado. Para justificar estadísticamente la restricción se aplica una prueba de significancia conjunta sobre todas las categorías asociadas a tal variable en la ecuación de resultado. Los resultados de la prueba que se presentan en la Tabla A-3 indican, con un nivel de significancia del $1 \%$, que omitir la variable es una restricción de exclusión válida. Los resultados del modelo HR se presentan en la Tabla 2.
La evidencia indica que, al aplicar la restricción de exclusión, no existe sesgo de selección en la asistencia a cine en Colombia. Lo anterior debido a que el coeficiente de correlación de los términos de error de las dos ecuaciones (rho) no es significativo y a que no se rechaza la prueba de Razón de Verosimilitud realizada para verificar la hipótesis nula de independencia entre las ecuaciones. Por tanto, cada ecuación se estima de manera independiente a través de un modelo probit y un modelo probit ordenado respectivamente. En este trabajo se interpretan los resultados de los últimos modelos que también se reportan en la Tabla 2. El lector puede verificar que las estimaciones no difieren en gran medida de las obtenidas en el modelo HNR en lo referente a magnitud, efectos marginales y significancia.

En lo concerniente a las características personales, los modelos estimados indican que la edad influye significativamente en la decisión de asistencia a cine y en la frecuencia con que los individuos realizan las visitas. Con respecto al segmento de población más joven (12-24 años), los individuos con edad en los rangos entre 2565 años y Más de 65 años tienen menor de probabilidad de pertenecer al grupo de asistentes. Dentro del grupo de asistentes, la probabilidad de pertenecer a las categorías de frecuencia superiores (Frecuente y Muy Frecuente) también es menor para los individuos en tales rangos de edad. El resultado, que coincide con lo reportado por Katsuura (2008), es el reflejo de la mayor disponibilidad de tiempo de los jóvenes y de la afinidad de esta población con los contenidos cinematográficos que los motiva a invertir parte de su tiempo de ocio en tal actividad. La probabilidad de asistencia para las mujeres es 1,89\% más alta que para los hombres; en la frecuencia de asistencia no se encontró un efecto significativo.

Los resultados del trabajo confirman que el capital cultural del individuo también tiene una influencia positiva sobre la participación en cine como lo demuestran Ateca-Amestoy (2010) y Muñiz, Rodríguez y Suárez (2017). En el caso del nivel educativo, por ejemplo, el efecto sobre la probabilidad de asistencia a cine es positivo y monótonamente creciente, es decir, la probabilidad de asistencia se incrementa con el nivel de 


\begin{tabular}{|c|c|c|c|c|c|}
\hline & Variable & $\begin{array}{l}\text { Muestra } \\
\text { completa }\end{array}$ & Asistentes & No asistentes & $\begin{array}{c}\text { Valor p } \\
\text { Prueba Chi-2 } \\
\text { de Pearson }\end{array}$ \\
\hline Sexo & $\begin{array}{l}\text { Masculino } \\
\text { Femenino }\end{array}$ & $\begin{array}{l}46,16 \% \\
53,84 \% \\
\end{array}$ & $\begin{array}{l}47,29 \% \\
52,71 \% \\
\end{array}$ & $\begin{array}{l}45,69 \% \\
54,31 \% \\
\end{array}$ & 0,02795 \\
\hline Edad & $\begin{array}{c}\text { 12-24 años } \\
25-65 \text { años } \\
\text { Más de } 65 \text { años }\end{array}$ & $\begin{array}{c}28,73 \% \\
61,75 \% \\
9,52 \%\end{array}$ & $\begin{array}{c}\mathbf{4 1 , 6 2} \% \\
56,56 \% \\
1,82 \%\end{array}$ & $\begin{array}{l}23,38 \% \\
63,90 \% \\
12,71 \%\end{array}$ & $<2,2 \mathrm{e}-16$ \\
\hline Estado Civil & $\begin{array}{c}\text { Soltero } \\
\text { Unión libre } \\
\text { Está casado(a) } \\
\text { Está separado(a) o } \\
\text { divorciado(a) } \\
\text { Está viudo(a) }\end{array}$ & $\begin{array}{c}37,12 \% \\
21,63 \% \\
22,86 \% \\
10,26 \% \\
5,13 \%\end{array}$ & $\begin{array}{c}\mathbf{5 3 , 4 4 \%} \\
17,08 \% \\
20,53 \% \\
7,69 \% \\
1,27 \%\end{array}$ & $\begin{array}{l}30,34 \% \\
27,76 \% \\
23,83 \% \\
11,33 \% \\
6,74 \%\end{array}$ & $<2,2 \mathrm{e}-16$ \\
\hline Etnia & $\begin{array}{l}\text { Mestizo(a) o Blanco(a) } \\
\text { Pertenece a alguna } \\
\text { comunidad étnica } \\
\text { (indígena, negro, gitano, } \\
\text { raizal o palenquero) }\end{array}$ & $\begin{array}{l}82,94 \% \\
17,06 \%\end{array}$ & $\begin{array}{c}\mathbf{8 8 , 6 3} \% \\
11,37 \%\end{array}$ & $\begin{array}{l}80,58 \% \\
\mathbf{1 9 , 4 2} \%\end{array}$ & $<2,2 \mathrm{e}-16$ \\
\hline Ocupación & $\begin{array}{c}\text { Trabajador } \\
\text { Busca trabajo } \\
\text { Estudiante } \\
\text { Oficios del hogar } \\
\text { Incapacitado } \\
\text { permanentemente para } \\
\text { trabajar } \\
\text { Otra }\end{array}$ & $\begin{array}{c}52,67 \% \\
3,43 \% \\
16,70 \% \\
20,77 \% \\
2,35 \% \\
\\
4,09 \%\end{array}$ & $\begin{array}{c}\mathbf{5 6 , 1 1 \%} \\
3,09 \% \\
\mathbf{2 6 , 9 0 \%} \\
10,46 \% \\
0,31 \%\end{array}$ & $\begin{array}{c}51,24 \% \\
3,57 \% \\
12,46 \% \\
\mathbf{2 5 , 0 5 \%} \\
\mathbf{3 , 2 0} \% \\
\\
\\
4,49 \%\end{array}$ & $<2,2 \mathrm{e}-16$ \\
\hline Ingreso & $\begin{array}{c}\text { Entre } \$ 0 \text { y } \$ 700.000 \\
\text { Entre } \$ 700.0001 \mathrm{y} \\
\$ 1.400 .000 \\
\text { Entre } \$ 1.400 .001 \mathrm{y} \\
\$ 2.100000 \\
\text { Entre } \$ 2.100 .001 \mathrm{y} \\
\$ 2.800 .000 \\
\text { Entre } \$ 2.800 .001 \mathrm{y} \\
\$ 4.200 .000 \\
\text { Más de } \$ 4.200 .000\end{array}$ & $\begin{array}{l}71,57 \% \\
18,21 \% \\
\\
4,32 \% \\
2,32 \% \\
2,22 \% \\
1,34 \%\end{array}$ & $\begin{array}{l}58,63 \% \\
\mathbf{2 2 , 1 6 \%} \\
\mathbf{7 , 0 3} \% \\
\mathbf{4 , 4 3} \% \\
\mathbf{4 , 5 1} \% \\
\mathbf{3 , 2 2} \%\end{array}$ & $\begin{array}{l}76,95 \% \\
16,57 \% \\
3,20 \% \\
1,44 \% \\
1,28 \% \\
0,56 \%\end{array}$ & $<2,2 \mathrm{e}-16$ \\
\hline Región & $\begin{array}{c}\text { Bogotá } \\
\text { Atlántica } \\
\text { Oriental } \\
\text { Central } \\
\text { Pacífica } \\
\text { Amazonía }\end{array}$ & $\begin{array}{l}17,06 \% \\
19,94 \% \\
17,30 \% \\
15,45 \% \\
17,48 \% \\
12,76 \%\end{array}$ & $\begin{array}{l}30,15 \% \\
14,94 \% \\
17,87 \% \\
13,66 \% \\
15,09 \% \\
8,28 \%\end{array}$ & $\begin{array}{l}11,63 \% \\
\mathbf{2 2 , 0 1} \% \\
17,06 \% \\
\mathbf{1 6 , 2 0} \% \\
\mathbf{1 8 , 4 8} \% \\
\mathbf{1 4 , 6 2} \%\end{array}$ & $<2,2 \mathrm{e}-16$ \\
\hline Nivel educativo & $\begin{array}{c}\text { Ninguno } \\
\text { Básica Primaria } \\
\text { Básica Secundaria } \\
\text { Media } \\
\text { Superior } \\
\text { Posgrado }\end{array}$ & $\begin{array}{l}5,33 \% \\
21,33 \% \\
21,34 \% \\
25,47 \% \\
23,41 \% \\
3,11 \%\end{array}$ & $\begin{array}{c}0,46 \% \\
4,75 \% \\
18,57 \% \\
26,09 \% \\
\mathbf{4 3 , 2 3} \% \\
\mathbf{6 , 9 0 \%}\end{array}$ & $\begin{array}{c}\mathbf{7 , 3 5 \%} \\
\mathbf{2 8 , 2 3} \% \\
\mathbf{2 2 , 5 0 \%} \\
25,21 \% \\
15,18 \% \\
1,54 \%\end{array}$ & $<2.2 \mathrm{e}-16$ \\
\hline $\begin{array}{c}\text { Prácticas en } \\
\text { audiovisuales }\end{array}$ & Sí asiste & $0,66 \%$ & $1,32 \%$ & $0,38 \%$ & 0,000833 \\
\hline $\begin{array}{c}\text { Talleres en } \\
\text { audiovisuales }\end{array}$ & Sí asiste & $0,22 \%$ & $0,52 \%$ & $0,09 \%$ & 0,008127 \\
\hline
\end{tabular}




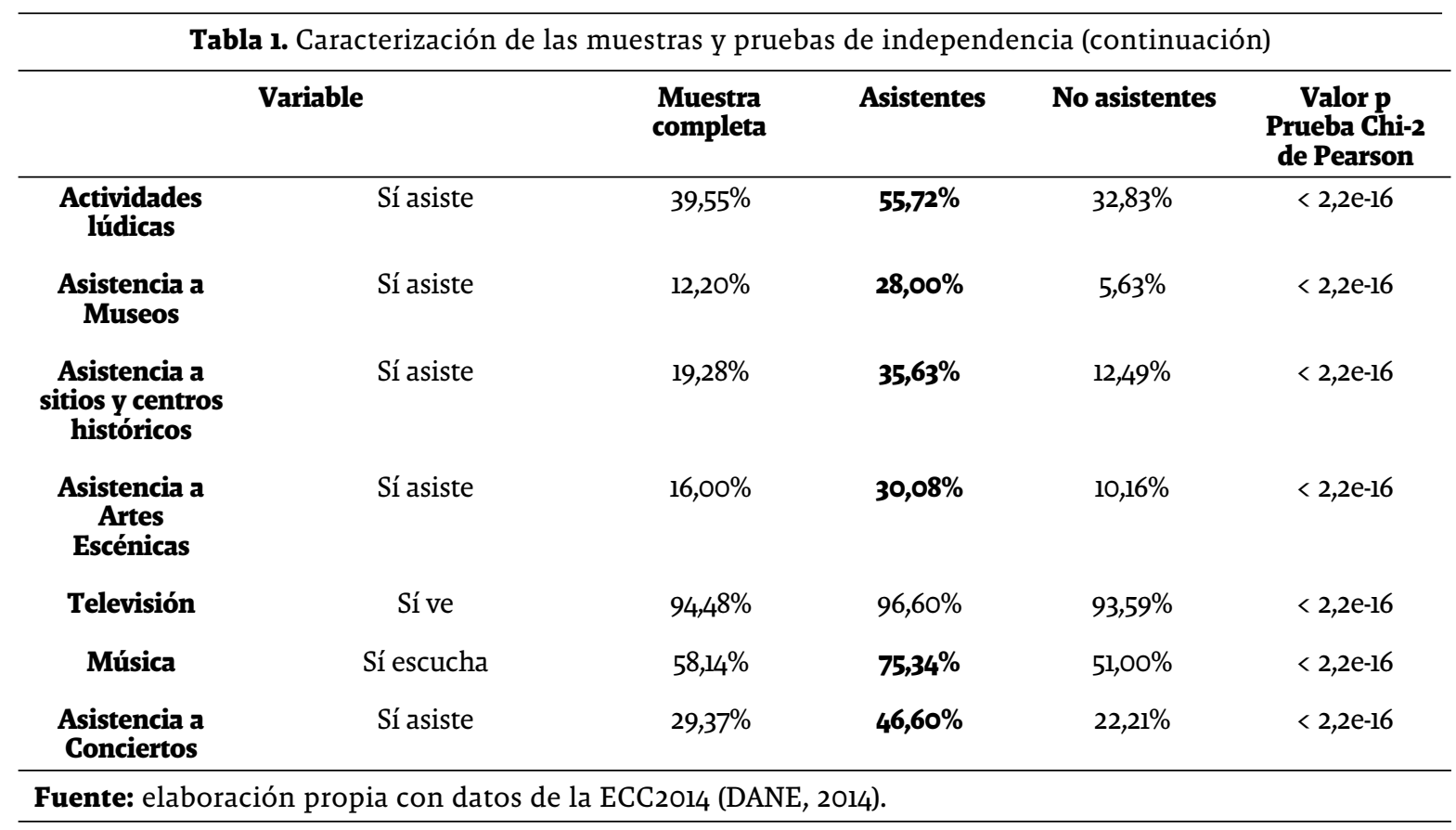

formación. Así, con respecto a los individuos sin educación, la probabilidad de pertenecer al grupo de asistentes es $16,45 \%, 28,34 \%$ y $31,64 \%$ más alta para quienes ostentan niveles de educación Media, Superior y Posgrado, respectivamente. En el modelo para la frecuencia de asistencia se observa un resultado similar: en los asistentes, la probabilidad de pertenecer a las categorías superiores de frecuencia es más alta para aquellos con mayor nivel educativo.

Las personas que participan en otras actividades del sector cultural como sitios de interés histórico, música, televisión, museos, artes escénicas y conciertos, tienen una mayor probabilidad de pertenecer al grupo de asistentes a cine. Acudir a museos, conciertos y sitios de interés histórico son las actividades con los efectos marginales más altos: $13,08 \%, 6,67 \%$ y $6,30 \%$ respectivamente. Realizar actividades lúdicas incrementa la probabilidad de pertenecer al grupo de asistentes en 4,26\%; en contraste, participar activamente en talleres y prácticas audiovisuales no es significativo ni para la asistencia ni para la frecuencia de asistencia a cine. Dentro del grupo de los asistentes, quienes participan en artes escénicas y/o realizan actividades lúdicas presentan una probabilidad mayor de pertenecer a las categorías de frecuencia más alta. En cambio, ver televisión está asociado a menos frecuencia de asistencia.

De los hallazgos anteriores es necesario comentar dos aspectos. En primer lugar, podría esperarse que la asistencia a cine compita con la asistencia a otras actividades del sector cultual en la asignación del tiempo de ocio; no obstante, la evidencia muestra una relación de complementariedad y no de sustitución, al menos en lo relacionado con la decisión de participación. Esto indica que los consumidores culturales en Colombia son "omnívoros" en la medida en que su consumo cultural se realiza sobre un espectro amplio y variado de productos culturales, característica evidenciada por otros autores en contextos no necesariamente similares al colombiano (Chan y Goldthorpe, 2007; Snowball, Jamas y Willis, 2010). En segundo lugar, el efecto del nivel educativo que se estima para la decisión y frecuencia de asistencia a cine es un efecto ceteris paribus que da cuenta de la importancia del nivel de conocimiento genérico sobre las decisiones de participación cultural; de tal efecto se aísla la posible influencia indirecta a través del canal indirecto de los ingresos 


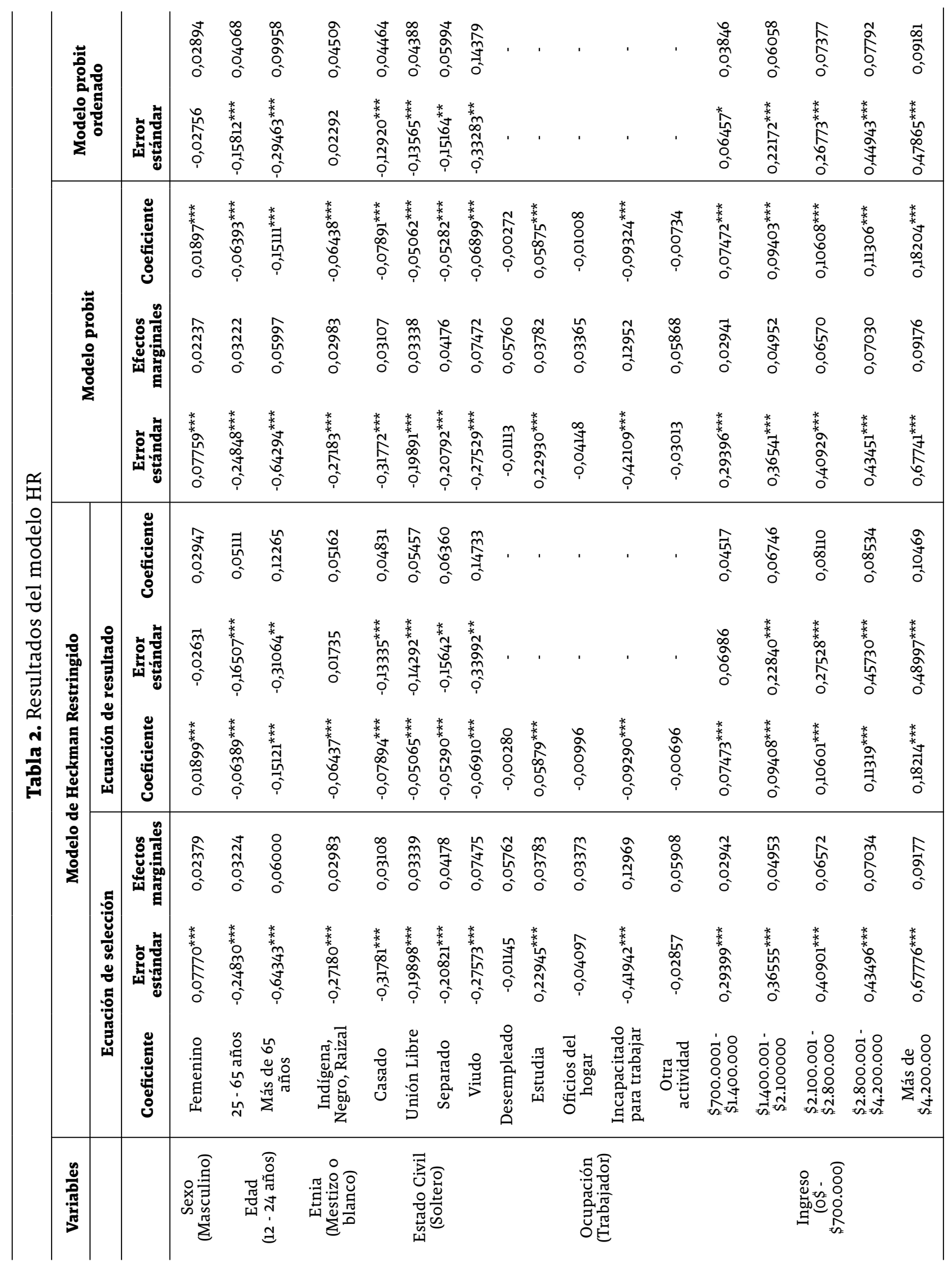




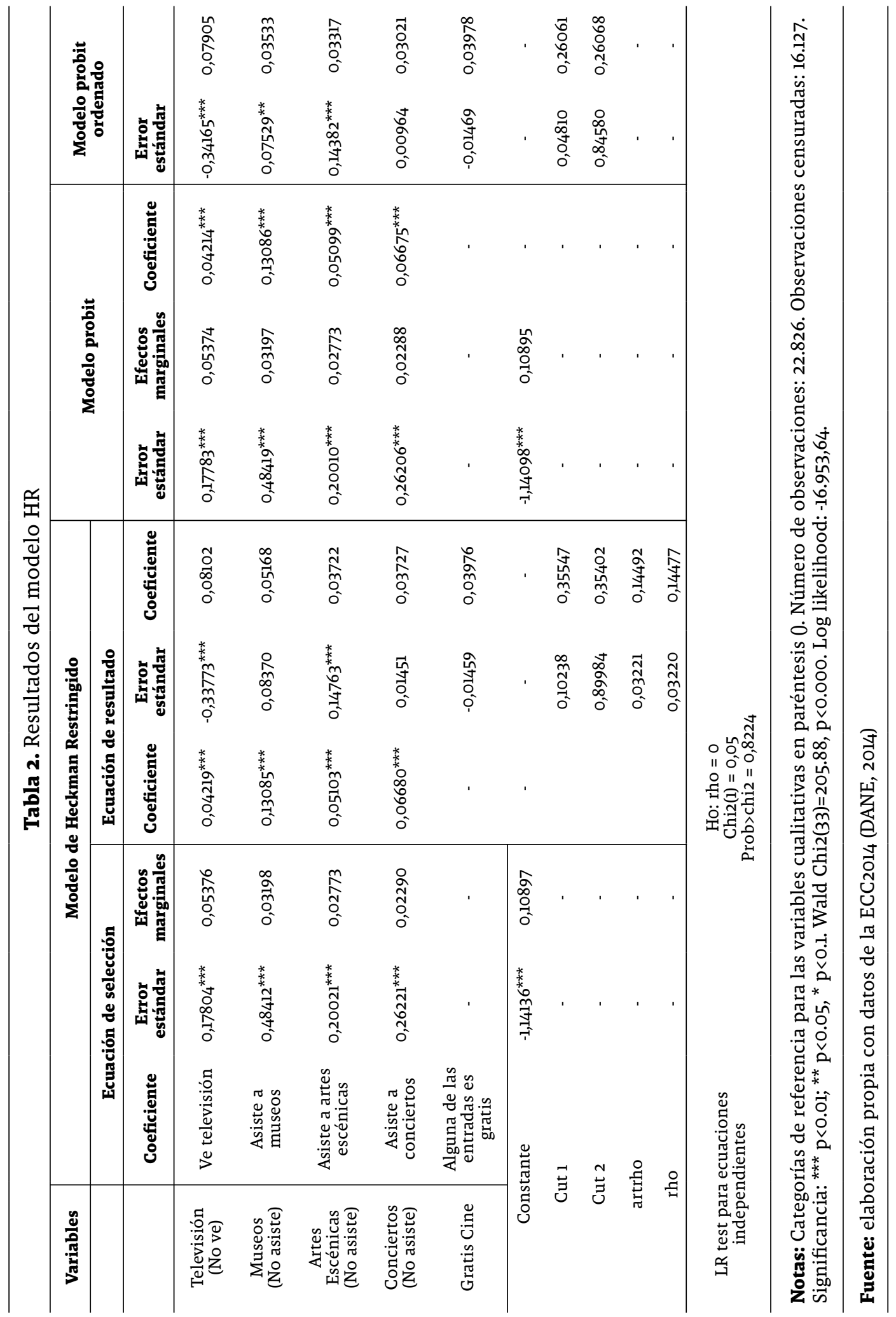


debido a que la última variable es considerada explícitamente como explicativa en el modelo.

Precisamente, los resultados muestran que el nivel de ingresos, al igual que lo reportado para el nivel de educación, tiene un efecto positivo y monótono sobre la decisión de asistencia a cine. Con respecto a los individuos con ingresos en el rango \$0-\$700.000, la probabilidad de pertenecer al grupo de asistentes se incrementa en $10,61 \%$ para quienes tienen ingresos entre \$2.100.001 y \$2.800.000; en $11,30 \%$ para quienes tienen ingresos entre $\$ 2.800 .001$ y $\$ 4.200 .000$, y en $18,20 \%$ para quienes tienen ingresos superiores a \$4.200.000. Comparando efectos marginales, es posible afirmar que el nivel educativo es mejor predictor que el nivel de ingresos, hallazgo que está en sintonía con los estudios empíricos revisados. En el modelo para la frecuencia de asistencia se observa que, con respecto a la población con ingresos más bajos, la probabilidad de pertenecer a las categorías de frecuencia de asistencia superiores es mayor para los individuos con ingresos altos.

Como era de esperarse, las restricciones de tiempo, capturadas en este estudio por las obligaciones familiares y laborales, tienen una influencia significativa sobre las dos variables dependientes de interés del estudio. Con respecto a los solteros, los individuos con cualquier otro estado civil tienen una probabilidad menor de pertenecer al grupo de asistentes; los efectos marginales más altos en valor absoluto están asociados a las categorías Casados $(-7,89 \%)$ y Viudos $(-6,89 \%)$. Similarmente, dentro del grupo de asistentes, las demás categorías del estado civil tienen una probabilidad menor de pertenecer a las categorías superiores de frecuencia. En el caso de la ocupación se observa que la probabilidad de asistencia para los estudiantes es 5,87\% más alta con respecto a los trabajadores; es importante recordar que esta variable no fue incluida en el modelo para la frecuencia de asistencia. Los resultados muestran que las menores responsabilidades familiares y laborales de los solteros y estudiantes facilitan la asistencia a cine, pues el costo de oportunidad del tiempo invertido en el consumo es menor para los individuos con tales características.
La Región de residencia del entrevistado también es un factor que influye en la decisión de asistir a cine y en la intensidad de la asistencia. Con respecto a Bogotá, la probabilidad de acudir es significativamente menor en las demás regiones del país: los efectos marginales se calcularon en $-20,84 \%,-16,22 \%,-14,16 \%,-12,51 \%$ y -12,43\% para las regiones Amazonía, Atlántica, Pacífica, Central y Oriental, respectivamente. De forma similar, los individuos asistentes que residen en regiones diferentes a Bogotá tienen menos probabilidad de pertenecer a las categorías de asistencia más alta. Tal resultado podría reflejar el efecto que tiene la mayor dotación de espacios para proyectar cine en la capital del país sobre las decisiones de asistencia, que reduce los costos en términos de tiempo y transporte para desplazarse al sitio de consumo. En efecto, según el Anuario Estadístico de Cine Colombiano (Mincultura, 2014), de las 879 salas activas en el país, el 29,8\% se ubican en Bogotá, mientras que solamente el 9,2\% están en Cali y el 8,4\% en Medellín. Las salas restantes están principalmente en ciudades capitales, mientras que la oferta en ciudades intermedias aún es incipiente.

Finalmente, el modelo para la frecuencia de asistencia indica que el hecho de que algunas entradas sean gratis no tiene un efecto significativo. Al analizar los datos se encuentra que el $15,49 \%$ de los asistentes asistió al menos una vez gratis; los porcentajes para cada categoría de frecuencia son: $16,87 \%$ para los ocasionales, $14,45 \%$ para los frecuentes y $14,83 \%$ para los muy frecuentes. El resultado refleja que la gratuidad de la entrada no es un fenómeno generalizado en Colombia debido a que en el país no existen políticas de formación de públicos para esta actividad; además, las entradas gratis que financian las instituciones que ofrecen el producto o las organizaciones culturales especializadas en el sector no alcanzan a un segmento importante de la población y no tienen un efecto determinante sobre las decisiones de consumo cultural. Es posible que la no existencia de este tipo de incentivos potencie el efecto barrera del nivel de ingresos sobre la participación, sin embargo, debido a la característica de los datos, tal hipótesis no puede verificarse en el presente trabajo. 


\section{Conclusiones}

En la definición de los patrones de consumo cultural de los individuos no solo intervienen las motivaciones personales, como lo plantean la sociología y la economía, sino también aspectos relacionados con los mecanismos de producción y distribución de los productos culturales. En el caso de los productos de las industrias culturales, y específicamente el cine, que son objeto de procesos de producción industrial y están inmersos en un modelo de consumo cultural de masas, se ha argumentado que los requerimientos de conocimientos y habilidades para ser disfrutados son menores que los requeridos para otro tipo de actividades como las artes escénicas o el patrimonio cultural, mientras que la influencia de otro tipo de factores como la edad, etnia o el género han mostrado ser más relevantes.

En el presente artículo se analizaron los determinantes de las decisiones individuales de asistencia y frecuencia de asistencia a cine en Colombia. Utilizando los microdatos de la ECC2014, aplicada por el DANE, se estimaron modelos de selección de Heckman para variable dependiente ordenada en los cuales la decisión de asistencia y la intensidad con que los individuos asisten fueron modeladas simultáneamente. Los resultados mostraron que no existen sesgos de selección muestral en el problema de estudio $y$, en consecuencia, los modelos probit y probit ordenados tradicionales también arrojan estimadores consistentes de los parámetros poblacionales. En la investigación se interpretaron los resultados de los modelos mencionados, aunque las estimaciones no difieren en gran medida en términos de magnitud, significancia y efectos marginales.

De los resultados se concluye, en primer lugar, que la tasa de asistencia a cine en Colombia es más alta que la reportada en otras actividades del sector cultural. Mientras que el 29,35\% de los individuos en la muestra manifiesta haber asistido al menos una vez a cine en el año anterior a la realización de la encuesta, este porcentaje es de apenas el $10,72 \%$ para los museos, $10,97 \%$ para bibliotecas, $18,18 \%$ para sitios de interés histórico (Ramos, 2017) y 16\% para cine, por mencionar solamente algunas actividades. En segundo lugar, se encontró que los determinantes de las decisiones de asistencia y frecuencia de asistencia a cine están en sintonía con los estudios sobre públicos citados desde la economía y la comunicación.

En efecto, se demostró que la edad es un factor que influye significativamente en las decisiones de consumo de cine de los colombianos. Con respecto al segmento de población más joven (12-24 años), los individuos en los rangos de edad más altos tienen menor probabilidad de asistir a cine y, de los que asisten, lo hacen con una menor frecuencia. En contraste, los solteros y los estudiantes asisten a más cine y con una frecuencia mayor, lo que responde al menor costo de oportunidad del tiempo invertido en el consumo cultural que implican las menores obligaciones familiares y laborales. Los hallazgos coinciden con lo que reportan los primeros estudios sobre públicos de cine realizados por Abrams (1950) y Katz y Lazarsfeld (1955), y refuerzan la idea del cine como una actividad social que llama la atención de los jóvenes porque motiva temas de conversación, facilita los contactos informales entre personas, promueve reuniones, citas, amistades $\mathrm{u}$ otras relaciones interpersonales o grupales como lo afirma Hinojosa (2016).

Aunque inicialmente se planteó la hipótesis de que los elementos del capital cultural tienen una menor relevancia para explicar las decisiones de consumo de cine, en este trabajo se encontraron efectos significativos y de magnitudes importantes tanto del nivel educativo como de la experiencia en otras actividades del sector cultural similares a los detectados por Katsuura (2008) y Ateca-Amestoy (2010). En el caso de la educación, se encontró que los individuos con mayores niveles de formación académica tienen más probabilidad de pertenecer al grupo de asistentes a cine, y además acuden con mayor frecuencia. La experiencia en otras actividades del sector cultural muestra una relación de complementariedad, pero solamente en la decisión de asistencia; en el caso de la frecuencia de asistencia los resultados no son concluyentes.

Sobre el último aspecto vale la pena destacar que, aunque la asistencia a cine es una actividad 
tiempo-intensiva, los resultados evidencian una relación de complementariedad con el consumo de otro tipo de actividades culturales como museos, teatros, sitios de interés histórico o música, que también requieren de tiempo para ser disfrutadas. El resultado se explica porque la mayor exposición de los consumidores a los productos culturales mejora el nivel de conocimiento y la capacidad de generar apreciación cultural, contribuyendo a la conformación de un grupo de consumidores culturales omnívoros que basan sus decisiones de consumo en un conjunto de elección más amplio y variado (Chan y Goldthorpe, 2007; Snowball et al., 2010). Tal aspecto podría ser investigado a profundidad en estudios posteriores.

En el país se han desarrollado algunas iniciativas de formación de públicos en cine. $\mathrm{La}$ Dirección de Cinematografía del Ministerio de Cultura cuenta con una línea de acción cuyo propósito es estimular y promover la circulación de contenidos audiovisuales y cinematográficos en el país a través de la difusión de obras nacionales, el fortalecimiento de muestras y festivales de cine y el apoyo a salas alternas. A pesar de tales iniciativas, en el estudio se evidencia la existencia de un efecto barrera de los ingresos que limita la asistencia a cine de los segmentos de población con menores recursos. En efecto, se encontró que la probabilidad de asistir a cine se incrementa con el nivel de ingresos y que, además, existe un efecto significativo de esta variable sobre la frecuencia de asistencia; adicionalmente, el hecho de que una de las entradas sea gratis no influye sobre la frecuencia de asistencia debido a que es un fenómeno poco generalizado que beneficia a aproximadamente el $15 \%$ de los asistentes. Una explicación es que los programas de formación de públicos se enfocan en promover la asistencia a salas alternas, mientras que la mayor parte de la población asiste a las salas comerciales, para las cuales no existe ningún tipo de estímulos; por tanto, el efecto de las políticas no se ve reflejado en los resultados del estudio.

Los resultados de esta investigación son de interés tanto para el sector público como para el sector privado, en la medida en que ofrece información sobre los perfiles de los demandantes de tal actividad, que es útil para la formulación de políticas de formación de públicos más focalizadas y para el diseño de estrategias comerciales que incrementen las ventas y maximicen los beneficios de los inversionistas de las salas comerciales. Algo especialmente interesante en un contexto en el que la participación de la población está creciendo, según lo reportan las diferentes mediciones realizadas por el DANE en la Encuesta de Consumo Cultural.

La información que ofrece la ECC2O14 presenta algunas limitaciones que se convierten en retos a enfrentar en futuras investigaciones. En primer lugar, agrega en una misma variable la asistencia a salas alternas y a salas comerciales. Una desagregación de la variable permitiría cuantificar el impacto de las políticas de estímulos en las decisiones de asistencia y establecer comparaciones entre los demandantes de los dos tipos de espacios. Segundo, la encuesta indaga sobre la asistencia a cine convencional, dejando de lado el consumo en plataformas audiovisuales y en la venta y alquiler de copias físicas que pueden ser incluso más masivos. Disponer de este tipo de información y triangularla con los datos que aporta el DANE permitiría ampliar la posibilidad de investigaciones para avanzar en el conocimiento de las características de los demandantes en el mercado de cine.

Tercero, sería deseable tener información sobre bienes y servicios complementarios que adquieren los consumidores durante las visitas a cine como comida, servicios de recreación, entre otros, lo cual permitiría una caracterización más profunda del perfil de los demandantes y la definición de estrategias comerciales.

Cuarto, la encuesta no indaga por el estrato socioeconómico, variable que según algunos estudios de participación cultural aplicados en Colombia (Aguado, 2011; Palma et al., 2014; Bermúdez et al., 2016; Espinosa y Toro, 2016; Gómez y Espinal, 2016), pueden explicar fenómenos de segregación espacial y de distinción que influyen en el comportamiento cultural de los consumidores. Finalmente, contar con variables como formación artística y cultural diferenciada 
por edades de inicio, si algún miembro de la familia pertenece al sector cultural, nivel de educación formal de los padres, experiencias de consumo previas, y satisfacción de las expectativas, permitiría caracterizar mejor el capital cultural de los individuos y verificar el cumplimiento de los diferentes enfoques teóricos del consumo cultural en el contexto colombiano.

\section{Anexos}

\begin{tabular}{|c|c|c|}
\hline Variable & Descripción & Tipo / Categorías \\
\hline \multicolumn{3}{|c|}{ Variables dependientes } \\
\hline Asistencia a Cine & $\begin{array}{l}\text { El individuo asistió a cine en el año } \\
\text { anterior a la encuesta. }\end{array}$ & $\begin{array}{c}\text { Binaria } \\
\text { l: Sí } \\
\text { o: No }\end{array}$ \\
\hline Frecuencia de asistencia & $\begin{array}{l}\text { Frecuencia de asistencia de los } \\
\text { individuos que sí asistieron a cine } \\
\text { en el año anterior a la encuesta. }\end{array}$ & $\begin{array}{l}\text { Categórica ordenada } \\
\text { l: Ocasionalmente } \\
\text { 2: Frecuentemente } \\
\text { 3: Muy frecuentemente }\end{array}$ \\
\hline \multicolumn{3}{|c|}{ Variables explicativas } \\
\hline \multicolumn{3}{|c|}{ Características Personales } \\
\hline Edad & Rango de edad del entrevistado. & $\begin{array}{l}\text { Categórica } \\
\text { 1: } 12-24 \text { años } \\
\text { 2: } 25-65 \text { años } \\
\text { 3: Más de } 65 \text { años }\end{array}$ \\
\hline Sexo & Sexo del encuestado. & $\begin{array}{l}\text { Categórica } \\
\text { l: Femenino } \\
\text { o: Masculino }\end{array}$ \\
\hline Estado Civil & Estado civil del encuestado. & $\begin{array}{c}\text { Categórica } \\
\text { 1: Soltero } \\
\text { 2: Casado } \\
\text { 3: Unión libre } \\
\text { 4: Separado o divorciado } \\
\text { 5: Viudo }\end{array}$ \\
\hline Etnia & $\begin{array}{l}\text { El encuestado se considera indígena, } \\
\text { negro, gitano, raizal o palenquero. }\end{array}$ & $\begin{array}{c}\text { Binaria } \\
\text { 1: Sí } \\
\text { o: No }\end{array}$ \\
\hline \multicolumn{3}{|c|}{ Características Socioeconómicas } \\
\hline Ingreso & $\begin{array}{l}\text { Nivel de ingresos mensual declarado } \\
\text { por el encuestado. }\end{array}$ & $\begin{array}{c}\text { Categórica } \\
\text { 1: Entre } \$ 0 \text { y } \$ 700.000 \\
\text { 2: Entre } \$ 700.001 \text { y } \$ 1.400 .000 \\
\text { 3: Entre } \$ 1.400 .001 \text { y } 2.100 .000 \\
\text { 4: Entre } \$ 2.100 .001 \text { y } \$ 2.800 .000 \\
\text { 5: Entre } \$ 2.800 .001 \text { y } \$ 4.200 .000 \\
\text { 6: Más de } \$ 4.200 .000\end{array}$ \\
\hline Ocupación & $\begin{array}{l}\text { Actividad económica en la que el } \\
\text { encuestado ocupó la mayor parte } \\
\text { del tiempo la semana anterior a la } \\
\text { encuesta. }\end{array}$ & $\begin{array}{c}\text { Categórica } \\
\text { 1: Trabajador } \\
\text { 2: Desempleado } \\
\text { 3: Estudiante } \\
\text { 4: Oficios del hogar } \\
\text { 5: Incapacitado permanente } \\
\text { 6: Otra actividad }\end{array}$ \\
\hline Personas & $\begin{array}{c}\text { Número de personas que viven en el } \\
\text { hogar. }\end{array}$ & Conteo \\
\hline
\end{tabular}


Tabla A-1. Descripción de las variables empleadas en el análisis (continuación)

\begin{tabular}{ccc}
\hline \multicolumn{2}{c}{ Tabla A-1. Descripción de las variables empleadas en el análisis (continuación) } \\
\hline Variable & Descripción & Tipo / Categorías \\
\hline \hline Nivel educativo & Características del Capital Cultural & \\
& Máximo nivel educativo alcanzado & Categórica: \\
& por el encuestado. & 1: Ninguno \\
& 2: Primaria \\
& 3: Secundaria \\
& $4:$ Media \\
& Universitaria) \\
& 5: Superióngica, \\
& 6: Posgrado (Especialización, \\
& Maestría, Doctorado).
\end{tabular}

Práctica en cine

Talleres en cine

Actividades lúdicas

Sitios y centros históricos

Escucha música

Televisión

Museos

Artes escénicas

Conciertos

Región
El encuestado realizó práctica en cine en el año anterior a la encuesta.

El encuestado realizó talleres en cine en el año anterior a la encuesta.

El encuestado asistió a actividades lúdicas en el año anterior a la encuesta.

El encuestado asistió a sitios de interés histórico en el año anterior a la encuesta.

El encuestado escuchó música en el año anterior a la encuesta.

El entrevistado vio televisión en el año anterior a la realización de la encuesta.

El encuestado asistió a museos en el año anterior a la encuesta.

El encuestado asistió a artes escénicas en el año anterior a la encuesta.

El encuestado asistió a conciertos en el año anterior a la encuesta.

\section{Ubicación geográfica}

Región en la que reside el encuestado.
Binarias

l: Sí

o: No

\section{Categórica \\ 1: Bogotá \\ 2: Atlántica \\ 3: Oriental \\ 4: Central \\ 5: Pacífica \\ 6: Amazonía}


Tabla A-2. Modelo HNR

Modelo de Heckman No Restringido

\begin{tabular}{|c|c|c|c|c|c|}
\hline \multicolumn{2}{|c|}{ Variables } & \multicolumn{2}{|c|}{ Ecuación de selección } & \multicolumn{2}{|c|}{ Ecuación de resultado } \\
\hline & & Coeficiente & Error estándar & Coeficiente & Error estándar \\
\hline $\begin{array}{c}\text { Sexo } \\
\text { (Masculino) }\end{array}$ & Femenino & $0,07801^{* * *}$ & 0,02239 & $-0,02004$ & 0,03068 \\
\hline $\begin{array}{c}\text { Edad } \\
(12-24 \text { años) }\end{array}$ & $\begin{array}{c}25-65 \text { años } \\
\text { Más de } 65 \text { años }\end{array}$ & $\begin{array}{l}-0,24801^{* * *} \\
-0,64296^{* * *}\end{array}$ & $\begin{array}{l}0,03225 \\
0,05997\end{array}$ & $\begin{array}{l}-0,17481^{* * *} \\
-0,28065^{* *}\end{array}$ & $\begin{array}{l}0,05123 \\
0,12565\end{array}$ \\
\hline $\begin{array}{c}\text { Etnia } \\
\text { (Mestizo o blanco) }\end{array}$ & $\begin{array}{c}\text { Indígena, Negro, } \\
\text { Raizal }\end{array}$ & $-0,27174^{* * *}$ & 0,02983 & 0,00847 & 0,05442 \\
\hline $\begin{array}{l}\text { Estado Civil } \\
\text { (Soltero) }\end{array}$ & $\begin{array}{c}\text { Casado } \\
\text { Unión Libre } \\
\text { Separado } \\
\text { Viudo }\end{array}$ & $\begin{array}{l}-0,31786^{\star * *} \\
-0,19894^{* \star *} \\
-0,20868^{\star * *} \\
-0,27631^{* \star *}\end{array}$ & $\begin{array}{l}0,03108 \\
0,03338 \\
0,04179 \\
0,07476\end{array}$ & $\begin{array}{l}-0,15003^{* * *} \\
-0,13272^{\star * *} \\
-0,16009^{\star *} \\
-0,34693^{* *}\end{array}$ & $\begin{array}{l}0,05567 \\
0,04861 \\
0,06366 \\
0,14786\end{array}$ \\
\hline $\begin{array}{l}\text { Ocupación } \\
\text { (Trabajador) }\end{array}$ & $\begin{array}{l}\text { Desempleado } \\
\text { Estudia } \\
\text { Oficios del hogar } \\
\text { Incapacitado } \\
\text { para trabajar } \\
\text { Otra actividad }\end{array}$ & $\begin{array}{l}-0,01097 \\
0,22939^{\star \star \star} \\
-0,04130 \\
-0,41978^{\star \star \star} \\
-0,03056\end{array}$ & $\begin{array}{l}0,05760 \\
0,03783 \\
0,03365 \\
0,12955 \\
0,05869\end{array}$ & $\begin{array}{l}0,03712 \\
-0,00809 \\
-0,05473 \\
-0,18824 \\
-0,18554^{* \star}\end{array}$ & $\begin{array}{l}0,08542 \\
0,05637 \\
0,05537 \\
0,26033 \\
0,08469\end{array}$ \\
\hline $\begin{array}{c}\text { Ingreso } \\
(0 \$ \$ \text { - } \$ 700.000)\end{array}$ & $\begin{array}{c}\$ 700.0001- \\
\$ 1.400 .000 \\
\$ 1.400 .001- \\
\$ 2.100000 \\
\$ 2.100 .001- \\
\$ 2.800 .000 \\
\$ 2.800 .001- \\
\$ 4.200 .000 \\
\text { Más de } \\
\text { \$4.200.000 }\end{array}$ & $\begin{array}{l}0,29377^{\star * *} \\
0,36560^{\star * *} \\
0,40830^{\star * *} \\
0,43546^{* * *} \\
0,67810^{* * *}\end{array}$ & $\begin{array}{l}0,02942 \\
0,04953 \\
0,06574 \\
0,07036 \\
0,09175\end{array}$ & $\begin{array}{l}0,06825 \\
0,22900^{* * *} \\
0,27621^{\star * *} \\
0,45777^{\star * *} \\
0,49329^{* * *}\end{array}$ & $\begin{array}{l}0,05348 \\
0,07345 \\
0,08727 \\
0,09091 \\
0,11213\end{array}$ \\
\hline Personas & $\begin{array}{l}\text { No. de personas } \\
\text { en el hogar }\end{array}$ & $-0,01136^{* *}$ & 0,00565 & $-0,01744^{* *}$ & 0,00844 \\
\hline $\begin{array}{l}\text { Región } \\
\text { (Bogotá) }\end{array}$ & $\begin{array}{l}\text { Atlántica } \\
\text { Oriental } \\
\text { Central } \\
\text { Pacífica } \\
\text { Amazonía } \\
\end{array}$ & $\begin{array}{l}-0,60880^{\star * *} \\
-0,45602^{* * *} \\
-0,45861^{\star \star *} \\
-0,52705^{* \star *} \\
-0,81343^{* * *}\end{array}$ & $\begin{array}{l}0,03441 \\
0,03353 \\
0,03504 \\
0,03440 \\
0,03886 \\
\end{array}$ & $\begin{array}{l}-0,09084 \\
0,17990^{* * *} \\
-0,24264^{* * *} \\
-0,13294^{*} \\
-0,54103^{* * *}\end{array}$ & $\begin{array}{l}0,07633 \\
0,06030 \\
0,06314 \\
0,06804 \\
0,09701 \\
\end{array}$ \\
\hline $\begin{array}{l}\text { Nivel Educativo } \\
\text { (Ninguno - } \\
\text { Preescolar) }\end{array}$ & $\begin{array}{l}\text { Primaria } \\
\text { Secundaria } \\
\text { Media } \\
\text { Superior } \\
\text { Posgrado }\end{array}$ & $\begin{array}{l}0,10123 \\
0,45822^{\star \star *} \\
0,72590^{\star * \star} \\
1,12175^{\star * *} \\
1,22406^{\star * \star}\end{array}$ & $\begin{array}{l}0,09088 \\
0,08989 \\
0,08872 \\
0,08949 \\
0,10592\end{array}$ & $\begin{array}{l}0,45983^{*} \\
0,61360^{\star *} \\
0,77730^{\star \star *} \\
1,10151^{\star \star *} \\
1,03285^{\star \star *}\end{array}$ & $\begin{array}{l}0,25396 \\
0,25849 \\
0,26758 \\
0,28616 \\
0,29725\end{array}$ \\
\hline $\begin{array}{l}\text { Práctica en } \\
\text { audiovisuales }\end{array}$ & $\begin{array}{l}\text { Realiza práctica } \\
\text { en audiovisuales }\end{array}$ & 0,01135 & 0,11633 & 0,17884 & 0,12907 \\
\hline $\begin{array}{l}\text { Talleres en } \\
\text { audiovisuales }\end{array}$ & $\begin{array}{l}\text { Realiza talleres } \\
\text { en audiovisuales }\end{array}$ & 0,20653 & 0,21044 & 0,13381 & 0,20510 \\
\hline $\begin{array}{l}\text { Actividades } \\
\text { Lúdicas }\end{array}$ & $\begin{array}{l}\text { Realiza } \\
\text { actividades } \\
\text { lúdicas }\end{array}$ & $0,17143^{* * *}$ & 0,02172 & $0,12607^{* * *}$ & 0,03485 \\
\hline $\begin{array}{c}\text { Sitios y Centros } \\
\text { Históricos }\end{array}$ & $\begin{array}{l}\text { Asiste a sitios de } \\
\text { interés histórico }\end{array}$ & $0,24543^{\star * *}$ & 0,02664 & 0,03946 & 0,04099 \\
\hline Escucha Música & Escucha música & $0,19495^{* * *}$ & 0,02282 & 0,03734 & 0,04054 \\
\hline Televisión & Ve televisión & $0,17835^{* * *}$ & 0,05376 & $-0,32948^{* * *}$ & 0,08236 \\
\hline
\end{tabular}


Nora Elena Espinal-Monsalve •Andrey David Ramos-Ramírez • Isabel Cristina Balbín-Espinal • Jorge Echavarría-Carvajal

\begin{tabular}{|c|c|c|c|c|c|}
\hline Museos & Asiste a museos & $0,48400^{* * *}$ & 0,03198 & $0,09914^{*}$ & 0,05688 \\
\hline \multicolumn{6}{|c|}{ Tabla A-2. Modelo HNR } \\
\hline & & \multicolumn{4}{|c|}{ Modelo de Heckman No Restringido } \\
\hline \multicolumn{2}{|c|}{ Variables } & \multicolumn{2}{|c|}{ Ecuación de selección } & \multicolumn{2}{|c|}{ Ecuación de resultado } \\
\hline & & Coeficiente & Error estándar & Coeficiente & Error estándar \\
\hline Artes Escénicas & $\begin{array}{l}\text { Asiste a artes } \\
\text { escénicas }\end{array}$ & $0,20004^{* * *}$ & 0,02773 & $0,15400^{* * *}$ & 0,03779 \\
\hline Conciertos & $\begin{array}{c}\text { Asiste a } \\
\text { conciertos }\end{array}$ & $0,26241^{* \star *}$ & 0,02290 & 0,02068 & 0,04042 \\
\hline \multicolumn{2}{|c|}{ Constante } & $-1,14148^{* * *}$ & 0,10898 & - & - \\
\hline \multicolumn{2}{|c|}{ Cut 1} & - & - & 0,17765 & 0,40404 \\
\hline \multicolumn{2}{|c|}{ Cut 2} & - & - & 0,97412 & 0,39873 \\
\hline \multirow{2}{*}{\multicolumn{2}{|c|}{$\begin{array}{l}\text { artrho } \\
\text { rho }\end{array}$}} & - & - & 0,08428 & 0,17699 \\
\hline & & - & - & 0,08408 & 0,17574 \\
\hline \multicolumn{3}{|c|}{ LR test para ecuaciones independientes } & \multicolumn{3}{|c|}{$\begin{array}{c}\text { Ho: rho }=0 \\
\text { Chi2(1) }=0,24 \\
\text { Prob }>\text { chi2 }=0,6233\end{array}$} \\
\hline
\end{tabular}

Tabla A-3. Prueba para restricciones de exclusión

Hipótesis Nula: Todos los coeficientes asociados a la ocupación son iguales a cero (0) conjuntamente.

Chi2 (5) $=6,19$

Prob $>$ chi2 $=0,2880$

Fuente: elaboración propia con datos de la ECC2014 (DANE, 2014).

\section{Referencias bibliográficas}

Abrams, M. (1950). The British cinema audience, 1949. Hollywood Quarterly, 4(3), 251-255. https://doi. org/10.2307/1209396

Aguado, L. (2011). Análisis empírico del comportamiento del consumidor de artes escénicas en Colombia (tesis doctoral). Universidad de Sevilla, Sevilla, España.

Aguado, L., Lazzaro, E., Palma, L. y Osorio, A. (2018). Newspaper reading as a form of cultural participation: The case of Colombia. Review of Economics and Finance, 11(1), 40-54.

Alonso, L. (1995). Los nuevos consumos culturales: ¿consumo de cultura o cultura de consumo? En Ministerio de Cultura (Ed.), Estado y cultura (pp. 153-196). Madrid, España: Ministerio de Cultura de España.

Andrade, D. (2016). Determinantes de la asistencia a museos en Colombia. Una perspectiva desde la economía de la cultura (tesis doctoral). Universidad de Sevilla, Sevilla, España.

Ateca-Amestoy, V. (2009). El capital humano como determinante del consumo cultural. Estudios de Economía Aplicada, 27(1), 87-110. 
Ateca-Amestoy, V. (2010). Cultural Participation Patterns: Evidence from the Spanish Time Use Survey. Recuperado de https://ssrn.com/abstract=1692024

Becker, G. y Murphy, K. (1988). A theory of rational addiction. Journal of Political Economy, 96(4), 675700. https://doi.org/10.1086/261558

Bermúdez, J. A., Medina, L. M. y Aguado, L. F. (2016). La decisión de escuchar música grabada en Colombia. Un enfoque microeconométrico. Revista de Métodos Cuantitativos Para La Economía y la Empresa, 21, 21-38.

Bocock, R. (1995). El consumo. Madrid, España: Talasa.

Borgonovi, F. (2004). Performing arts attendance: an economic approach. Applied Economics, 36(17), 1871-1885. https://doi.org/10.1080/0003684042000264010

Bourdieu, P. (1984). Distinction. A Social Critique of the Judgement of Taste. Cambridge, Estados Unidos: Harvard University Press. https://doi.org/10.4324/9781315680347-10

Cameron, S. (1999). Rational addiction and the demand for cinema. Applied Economics Letters, 6(9), 617-620. https://doi.org/10.1080/135048599352736

Castiglione, C. e Infante, D. (2016). Rational addiction and cultural goods: the case of the Italian theatregoer. Journal of Cultural Economics, 40(2), 163-190. https://doi.org/10.1007/s10824-015-9247-4

Chan, T. y Goldthorpe, J. (2007). Social stratification and cultural consumption: Music in England. European Sociological Review, 23(1), 1-19. https://doi.org/10.1093/esr/jclo16

DANE -Departamento Nacional de Estadística-. (2014). Encuesta de Consumo Cultural. Recuperado de http://microdatos.dane.gov.co/index.php/catalog/345/get_microdata

De Luca, G. y Perotti, V. (2011). Estimation of Ordered Response Models With Sample Selection. The Stata Journal, 11(2), 213-239. https://doi.org/10.1177/1536867X1101100204

Espinosa, A. y Toro, D. (2016). La participación en la vida cultural en Cartagena, 2008-2013. Economía y Región, 10(1), 217-248.

Falk, M. y Falk, R. (2011). An ordered probit model of live performance attendance for $24 \mathrm{EU}$ countries. Austrian Institute of Economic Research (WIFO), 1-25. Recuperado de http://www.siecon.org/ online/wp-content/uploads/2011/o4/Falk-Falk1.pdf

Fernández-Blanco, V. y Prieto-Rodríguez, J. (2009). Análisis de los hábitos de lectura como una decisión económica. Estudios de Economía Aplicada, 27(1), 87-110.

García, N. y Piedras, E. (2000). Las industrias culturales y el desarrollo de México. Ciudad de México, México: Siglo XXI Editores.

Gómez, L. y Espinal, N. (2016). Determinantes de la participación en artes escénicas en Medellín, Colombia. Ensayos de Economía, 26(48), 189-207. https://doi.org/10.15446/ede.v26n48.59863

Graziano, P., Escobar, A., Angulo, G. y Mercado, M. (2017). Participación en actividades culturales y recreativas en la Región Atlántica de Colombia (2016). Recuperado de https://www.mincultura.gov. co/areas/cinematografia/publicaciones/Documents/Anuario\%20Estad\%C3\%ADstico\%20Cine\%20 Colombiano\%202014.pdf 
Nora Elena Espinal-Monsalve • Andrey David Ramos-Ramírez • Isabel Cristina Balbín-Espinal • Jorge Echavarría-Carvajal

Greene, W. y Hensher, D. (2010). Modeling ordered choices: A primer. Cambridge, Reino Unido: Cambridge University Press. https://doi.org/10.1017/CBO9780511845062

Heckman, J. (1979). Sample selection bias as a specification error. Econometrica, 47(1), 153-161. https:// doi.org/10.2307/1912352

Herrera-Usagre, M. (2010). Estratificación social y estilos de vida culturales. Documentos de trabajo (Centro de Estudios Andaluces), 2(4), 1-53.

Hinojosa, L. (2016). Cine, memoria y ciudad: el estudio de los públicos de cine en las Ciencias Sociales. Opción: Revista de Ciencias Humanas y Sociales, 32(13), 492-513.

Jenks, C. (1993). Culture. Londres, Reino Unido: Routledge. https://doi.org/10.4324/9780203446317

Katsuura, M. (2008). Examining arts participation in Japan using the survey on time use and leisure activities. Asia Pacific Journal of Arts and Cultural Management, 5(1), 343-361.

Katz, E. y Lazarsfeld, P. (1955). Personal Influence. Glencoe, Escocia: Free Press. https://doi.org/ $10.4324 / 9781315126234$

Keane, M. (1992). A note on identification in the multinomial probit model. Journal of Business and Economic Statistics, 10(2), 193-200. https://doi.org/10.1080/07350015.1992.10509898

Lazcano, I. y Landabidea, X. (2010). El consumo cultural y la creación de audiencias escénicas a través de la participación en eventos escénicos extraordinarios. En M. Cuenca, I. Lazcano y X. Landabidea (Eds.), Sobre ocio creativo: situación actual de las Ferias de Artes Escénicas (pp. 4972). Bilbao, España: Universidad de Deusto. Recuperado de http://www.deusto-publicaciones.es/ deusto/pdfs/ocio/ocio41.pdf

Lee, L. (1995). Semiparametric maximum likelihood estimation of polychotomous and sequential choice models. Journal of Econometrics, 65(2), 381-428. https://doi.org/10.1016/0304-4076(93)01591-9

Lévy-Garboua, L. y Montmarquette, C. (1996). A microeconometric study of theatre demand. Journal of Cultural Economics, 20(1), 25-50. https://doi.org/10.1007/s10824-005-5050-y

Meng, C. y Schmidt, P. (1985). On the cost of partial observability in the bivariate probit model. International Economic Review, 26(1), 71-85. https://doi.org/10.2307/2526528

Mincultura -Ministerio de Cultura-. (2014). Anuario Estadístico de Cine Colombiano. Recuperado de https://www.mincultura.gov.co/areas/cinematografia/publicaciones/Documents/Anuario\%20 Estad\%C3\%ADstico\%20Cine\%20Colombiano\%202014.pdf

Muñiz, C., Rodríguez, P. y Suárez, M. (2017). Participation in cultural activities: specification issues. Journal of Cultural Economics, 41(1), 71-93. https://doi.org/10.1007/s10824-015-9261-6

Palma, M. L., Aguado, L. y Osorio, A. M. (2014). Determinants of book reading and library attendance in Colombia. A microeconometric approach. Economics and Business Letters, 3(2), 79-86. https:// doi.org/10.17811/ebl.3.2.2014.79-86

Ramos, A. (2017). Consumo de bienes del Patrimonio Cultural en Colombia: análisis microeconómico de los determinantes desde los modelos de elección discreta regularizados (tesis de maestría). Universidad Nacional de Colombia, Medellín, Colombia. 
Sisto, A. y Zanola, R. (2010). Cinema attendance in Europe. Applied Economics Letters, 17(5), 515-517. https://doi.org/10.1080/13504850802046997

Snowball, J., Jamal, M. y Willis, K. (2010). Cultural consumption patterns in South Africa: An investigation of the theory of cultural omnivores. Social indicators research, 97(3), 467-483. https:// doi.org/10.1007/s11205-009-9513-y

Stigler, G. y Becker, G. (1977). De gustibus non est disputandum. The American Economic Review, 67(2), 76-90.

Thompson, J. (1990). Ideology and Modern Culture. Stanford, Estados Unidos: Stanford University Press.

Throsby, D. (1994). The production and consumption of the arts: A view of cultural economics. Journal of Economic Literature, 32(1), 1-29.

Tudor, A. (1974). Cine y comunicación social. Barcelona, España: Gustavo Gili/Comunicación Visual.

Varela, F. (1988). El círculo creativo. Esbozo histórico natural de la reflexibilidad. En P. Watzlawick (Ed.), La realidad inventada (pp. 251-263). Buenos Aires, Argentina: Gedisa. 